Re: Comparison of 2 Transvaginal Surgical Approaches and Perioperative Behavioral Therapy for Apical Vaginal Prolapse: The OPTIMAL Randomized Trial

Barber MD, Brubaker L, Burgio KL, et al

JAMA 2014;311:1023-34

\section{Experts' summary:}

This well-designed, multicentre, randomised trial evaluated a very interesting and clinically relevant topic: the comparison between two different transvaginal surgical techniques to repair apical vaginal prolapse. Moreover, the authors assessed the impact of the behavioural therapy and the pelvic floor muscle training on the outcomes of these surgical procedures. In the present paper, a total of 374 patients with apical prolapse, with or without concomitant prolapse of the other vaginal compartment, were enrolled in the study: 188 in the uterosacral ligament vaginal vault suspension (ULS) group and 186 in the sacrospinous ligament fixation (SSLF) group. The cure rate was assessed at 24 mo using a very strict and rigorous definition of overall surgical success. At 2-yr followup, no differences were found in terms of anatomic, functional, or adverse-event outcomes of the two groups. The overall surgical success rate was $59.2 \%$ for USL and $60.5 \%$ for SSLF. In addition, the authors observed that behavioural therapy and pelvic floor muscle training did not influence the surgeryrelated outcomes of these two procedures.

\section{Experts' comments:}

In the past decade, the vast majority of the published trials on surgical treatment of pelvic organ prolapse, in particular of apical vaginal prolapse, have considered only procedures using synthetic graft materials. Some reviews and metaanalyses on this issue concluded that sacrocolpopexy (performed via open abdominal, laparoscopic, or robot-assisted routes) represents the gold standard in the surgical treatment of vaginal vault prolapse, ensuring superior anatomic outcomes to procedures performed via the vaginal route (including sacrospinous colpopexy and uterosacral colpopexy) $[1,2]$. However, although precise estimates are not available, most studies suggest that the vaginal approach is most common, with $80-90 \%$ of procedures being performed via this route [3].

The trial by Barber et al. has the great merit of focusing attention, after so many years, on the role of native-tissue vaginal reconstructive surgery for the treatment of apical prolapse. In the actual scenario of general revision of mesh utilisation, it could be clinically important to detect an effective and safe surgical reconstructive vaginal procedure for the correction of apical vaginal compartment prolapse. Unfortunately, very few data with medium- to long-term follow-up still exist.

In the present study, Barber et al. reported overall surgical success of $60 \%$. Although the success rate reported in this series is lower than success rate reported in the literature (about $70-90 \%$ ), this series considered a very rigorous definition of cure. In fact, at 24 mo of follow-up, only $16-20 \%$ of patients reported prolapse symptoms and, even more relevant, $<5 \%$ of women required retreatment.

This excellent study presents the same weakness as the majority of the papers on surgery of pelvic organ prolapse, namely, the relatively short follow-up. In 1966, TeLinde stated, "Every honest surgeon of extensive and long experience will have to admit that he is not entirely and absolutely satisfied with his long-term results of all his operations for prolapse and allied conditions" [4]. Barber et al have shown the way. Now this topic requires several other randomised trials with medium- and long-term follow-up.

Conflicts of interest: The authors have nothing to disclose.

\section{References}

[1] Maher C, Feiner B, Baessler K, Schmid C. Surgical management of pelvic organ prolapse in women. Cochrane Database Syst Rev 2013:CD004014.

[2] Serati M, Bogani G, Sorice P, et al. Robot-assisted sacrocolpopexy for pelvic organ prolapse: a systematic review and meta-analysis of comparative studies. Eur Urol 2014;66:303-18.

[3] Barber MD, Maher C. Apical prolapse. Int Urogynecol J 2013;24: 1815-33.

[4] TeLinde RW. Prolapse of the uterus and allied conditions. Am J Obstet Gynecol 1966;94:444-63.

Maurizio Serati*, Giorgio Bogani, Paola Sorice, Maria Carmela Di Dedda, Fabio Ghezzi Department of Obstetrics and Gynecology, University of Insubria, Varese, Italy

${ }^{*}$ Corresponding author. Department of Obstetrics and Gynecology, Urogynecology Unit, University of Insubria, Piazza Biroldi 1, 21100 Varese, Italy. E-mail address: mauserati@hotmail.com (M. Serati).

http://dx.doi.org/10.1016/j.eururo.2014.09.044

\section{Re: Risk Of Incisional Hernia After Minimally Invasive and Open Radical Prostatectomy}

Carlsson SV, Ehdaie B, Atoria CL, Elkin EB, Eastham JA

J Urol 2013;190:1757-62

\section{Experts' summary:}

This population-based study compared the incidence of incisional hernia $(\mathrm{IH})$ repairs for open prostatectomy $(\mathrm{OP})$ and robot-assisted radical prostatectomy (RARP). Carlsson and colleagues demonstrated in a large Surveillance Epidemiology and
End Results-Medicare population-based study that the incidence of IH repair is much higher following RARP than previously suggested (5.3\%) and much higher than for OP (1.9\%). After adjusting for several characteristics, the adjusted hazard ratio was 3.39 (95\% confidence interval, $2.63-4.38 ; p<0.0001$ ). The authors attribute the findings to surgical technique.

\section{Experts' comments:}

IH is a significant complication of RARP that warrants specific consideration. Clearly, the risk of developing a symptomatic 